\title{
New Systems Solution for Resolving Nonlinear Dynamics Based on Systems Thinking
}

\author{
Deok-soo Cha', Kyoung-il Kim² \\ ${ }^{1}$ Eho Technology Co. Seoul, S. Korea, Soong-Sil University, Seoul, Korea \\ ${ }^{2}$ Electrical Power Division, Seoul in S. Korea, Chung-Ang University, Seoul, Korea \\ Email: chdsoo@hotmail.com, kb01155@naver.com
}

How to cite this paper: Cha, D.S. and Kim, K.I. (2021) New Systems Solution for Resolving Nonlinear Dynamics Based on Systems Thinking. Open Journal of Applied Sciences, 11, 1177-1189.

https://doi.org/10.4236/ojapps.2021.1110088

Received: September 19, 2021

Accepted: October 26, 2021

Published: October 29, 2021

Copyright $\odot 2021$ by author(s) and Scientific Research Publishing Inc. This work is licensed under the Creative Commons Attribution International License (CC BY 4.0).

http://creativecommons.org/licenses/by/4.0/

\begin{abstract}
This study describes a new solution for resolving nonlinear dynamics. Surprisingly, it has been resolved and completed by non-physicists on behalf of physicists in 2021. It is a revolutionary solution like the Copernican Theory, which is perfectly different from the existing chaos theory. In the past, nonlinear dynamics has been analyzed using logical solutions, such as chaos theory, based on logical thinking. However, it is not perfect systematic solution, hence; the new solution has been analyzed and resolved by systematic analytical tool in other sciences. Then, the result is more perfect and precise than the old chaos theory. Regrettably, most physicists do not welcome this advancement, because they have primitive solutions such as chaos theory. If the new solution is true, it is very disadvantageous to them like Galileo's heliocentric theory. Therefore, they do not welcome it and deny and reject it. Hence, they wish it to fail; moreover, they want to remain in safe zone. Unfortunately, they became outsiders because they have no ability to review new solutions. Unfortunately, we have no obligation to follow physicists. If so, non-physicists, bypassing physicists, must study independently nonlinear dynamics based on systems thinking, and have to share the findings other scientists. It means that the new solution would be replaced the chaos theory in traditional physics; moreover, it would be resolved many unsolved nonlinear dynamics in the future.
\end{abstract}

\section{Keywords}

Nonlinear Dynamics, Control Theory, MATLAB, Systems Thinking

\section{Introduction}

This article details an innovative solution for resolving nonlinear dynamics involving complexity [1], such as ecosystems or thermodynamic systems. Remarkably, 1) this systematic solution has been resolved by non-physicists on behalf of 
physicists and 2) it has been studied through interdisciplinary research between physics and engineering science for the first time. 3) Regrettably, it will not be advantageous the physicists. Hence, the readers keep in mind the above three description. In this article, it will be provided the reason to non-physicists and physicists.

Meanwhile, in modern science, there is a systematical solution such as chaos theory, which is arranged using algebra and statistics by classical physicists in the $17^{\text {th }}$ century; they approached the systematical problems with static macroscopic viewpoints based on logical thinking as shown in Figure 1(b). And then, physicists treated the food chain in ecosystems as a logical problem based on logical thinking. Since most determinists did not distinguish between logical thinking and systems thinking (refer to Section 2.3). Nevertheless, they found was that its external behavior of systems has complexity including irregularity, regularity, self-organization and initial phenomena. Thus, it is a serious mistake! Why do it? Because they have no idea about the paradigm of systems thinking. Therefore, they have no perfect systematic solution until now. Unfortunately, no determinists do know this problem.

Surprisingly, in 2021, a non-physicist has found the perfect solution for resolving nonlinear dynamic systematic problems such as ecosystems or thermodynamic systems as shown in Table 1 on behalf of physicists. It is a shocking scientific result because they prove that there is a perfect solution in science. If it is true, it is similar to the heliocentric theory of medieval Galileo. Physicists as well as non-physicists know what this means. This is a very interesting research result for non-physicists if they are not fool. Otherwise, physicists have no choice. They should not be silent about this solution.

However, we have a question how can achieve the solution. First of all, the readers watch the Figures 1(a)-(c). The researcher defined that nonlinear dynamics such as ecosystems as shown in Figure 1(b) and Figure 1(c) is not treated as a black box but a closed loop system, as shown in Figure 2. And then, they succeed in analyzing the ecosystem using the systems analytic method [2] as shown in Figure 1(a). Nevertheless, no physicists know the analyzing theory. Unfortunately, the analyzing method did not exist in classical physics in the $17^{\text {th }}$ century. Therefore, it must be studied via interdisciplinary research between physics and engineering; it is need to introduce the analyzing method into physics. However, physicists avoid cooperation with engineering.

Table 1. Comparison of the old logical solution and new systematical solution for closed loop systems in modern science (More detail of new solution will be described in Section 2).

\begin{tabular}{lll}
\hline Division & Old Logical Solution & New Systematical Solution \\
\hline Paradigm of thinking & Logical thinking based on Determinism & $\begin{array}{l}\text { Systems thinking based on } \\
\text { Indeterminism + (Determinism) } \\
\text { How to solve logical problem }\end{array}$ \\
How to solve systematical problem & Solved by algebra in traditional physics & The same as left solution \\
& Solved by Chaos theory based on logical thinking & $\begin{array}{l}\text { Solved by systems analysis theory based } \\
\text { on systems thinking }\end{array}$ \\
\hline
\end{tabular}



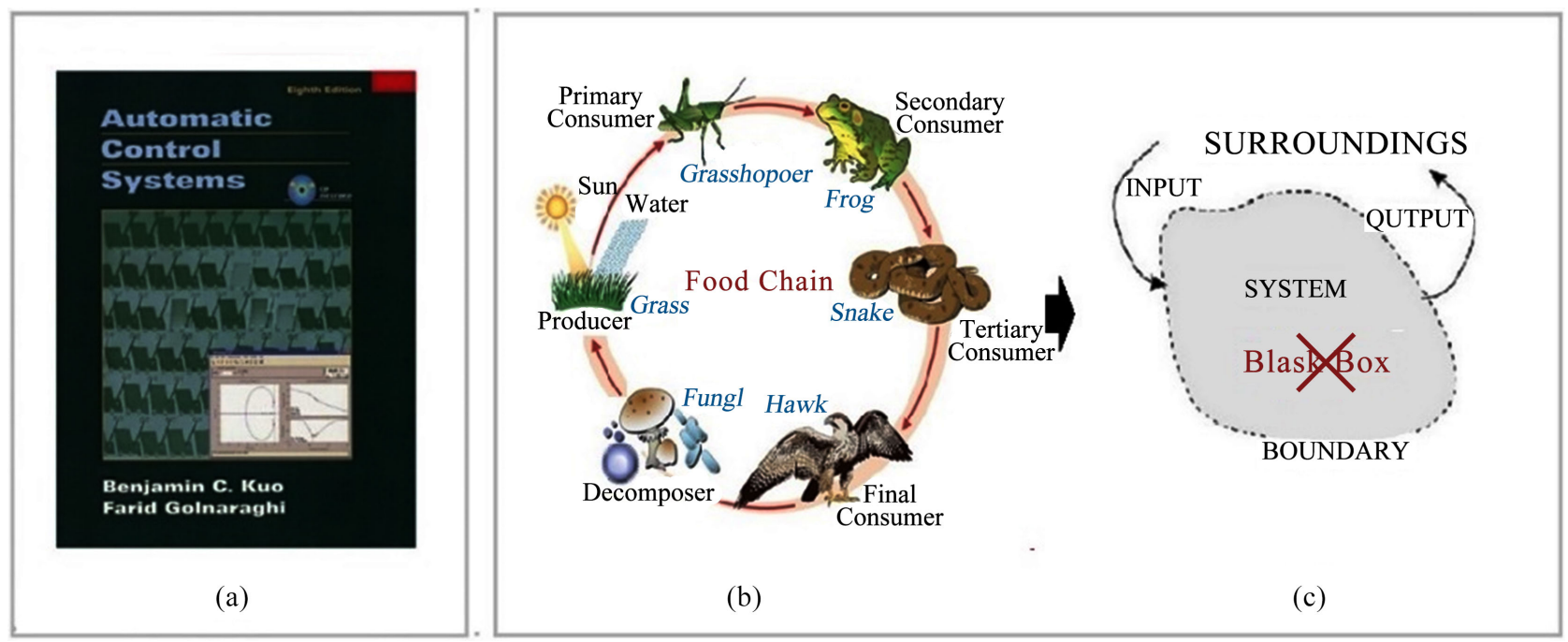

Figure 1. (a) Textbook on control theory; (b) Food chain in ecosystems; (c) Basic system. [Remark; the food chain in (b) in nature is exactly a eternal circulatory system.].
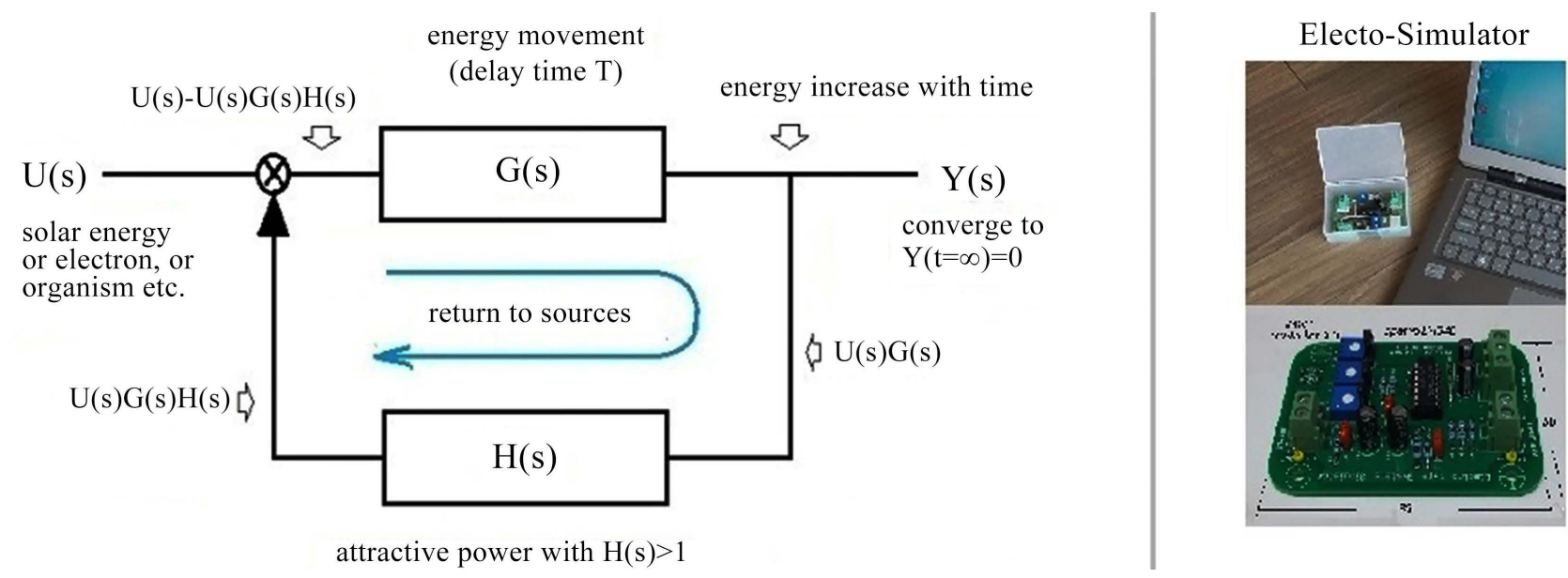

Figure 2. Internal structure of closed loop systems in nature and analog electro simulator with the same characteristics. [Remark; Entropy G(s), such as the desire to propagation, must be returning to the source with a delay time d, because it is based on the law of conservation of energy, and macroscopically, the output finally converges to zero; it is theoretical background.

Amazingly, as expected, most physicists do not welcome and strongly deny and reject because it is very dangerous result to them like medieval Copernican theory. They have to abandon their chaos theory, besides, they have a risk losing everything they are like medieval astronomers; moreover, physicists have made a mistake with the public for the past 300 years. In addition, they have no ability to review and evaluate the new solution because they have not learned the analyzing method. Ironically, they misunderstood the above solution as epistemological problem; it is nonsense. In addition, they require the author to present perfect evidence that the chaos theory is imperfect, it means that they keep in using the Ptolemaic theory; it is the same as nonsense because they misunderstood that they are outsiders.

Meanwhile, the author's mission is to persuade the physicists and to inform the emergence of these solutions for non-physicists. For this, the author will pre- 
sent the perfect evidence they required in Section $2.5 ; 1$ ) the famous Lorenz's butterfly effect, 2) random walks in the stock market, 3) the logistic curve in ecosystems, and 4) Kuhn's innovation theory. By the way, the author is intended to be informed this solution to non-physicists. We expect the rational judgment of non-physicists.

\section{Materials and Methods}

\subsection{Scientific Background}

The new solution in Table 1 has not been resolved by physicists. It has been resolved via interdisciplinary research between physics and engineering by non-physicists. How can they do so? As shown in Table 1, it is compared with chaos theory in left side. The right side of the Table 1 describes the new solution as mentioned above for resolving nonlinear dynamics. Amazingly, any physicists has not ever seen before because it has resolved using the system analytical method as shown in Figure 1(a).

The author attempted to introduce the above analytical method into nonlinear dynamics: it means $\{$ Control theory $\} \rightarrow\{$ Physical science $\}$. And then, the author succeeded in resolving the nonlinear dynamics such as food chain in ecosystems, as shown in Section 2.5. Moreover, the readers refer to the report [3] [4]. Unfortunately, it is outside of current academic framework of determinism. Accordingly, most determinists who adhere determinism do not acknowledge the new solution but there is no problem. However, non-physicists have no obligation to follow them if they want to remain outsiders who wish the author to fail and invalidate the new solution. Again, physicists have no choice, moreover, they are not outsiders.

On the other hand, we need to investigate why determinists do not know the paradigm of scientific thinking. For instance, it is classified into \{logical thinking\} and \{systems thinking\} [5]. Unfortunately, classical physicists have no idea on systems thinking because their determinism; it has not used the systems analysis theory at all. For instance, the author received an opinion from a physicist in physics journal, S, in 2021. Ironically, they accepted this solution as an epistemological problem; it is nonsense; it implies that they are outriders moreover, they have no the ability to review and evaluate the solution in Table 1.

In contrast, if non-physicists have no reason to object on the solution because it is a more advanced solution than the old chaos theory can be applied to high-tech industries such as artificial intelligence (AI). While the new solution is perfect without doubt, non-physicists would not trust them and their statistical physics including chaos theory. In special, the new solution has been proved by experimental using MATLAB program [6] or simulator, or a novel analog simulation device as shown in Figure 2 in right side. Anyone can confirm its validity using the devices. Unfortunately, if determinists avoid confirming the result, they are like medieval astronomers; moreover, they want to remain outsiders to stay in safe zone. 


\subsection{Theoretical Background}

There are many unsolved problems in nonlinear dynamic systems, such as ecosystems turbulence, climate change, and organisms in modern science. Why can classical physicists not resolve the problems? Unfortunately, they have no solution for resolving closed loop systems with feedback mathematically, hence, they resolved both logical and systematic problems using the same logical solution for a long time. Hence, they treated a representative systematic problem such as ecosystems as a black box as shown in as shown in Figure 1(b) and Figure 1(c). Unfortunately, it is not their mistake because the above-mentioned system analytical theory has been developed in the $20^{\text {th }}$ century past 300 years by engineering scientists. However, even they cannot avoid their responsibility.

Nevertheless, they are remained outsiders and they stay safe zone; other scientists need not cooperated with them. Therefore, this section will describe reason to non-physicists. In long time ago, the author has discovered an incomprehensible problem that physicists have no perfect systematic solution based on systems thinking; it is a serious mistake in physics. Amazingly, it is shocking that physicists do not know that systems analyzing theory as shown in Figure 1(a) exist in other science. What is the systems analyzing theory? Physicists have not known it.

Generally, the analytical method was developed for designing automatic control systems during the Second World War; moreover; it is widely used as an engineering principle and other science except physics. Paradoxically, if classical physicists had known the above analyzing theory, they would have succeeded in resolving many unsolved problems including metaphysical problems such as "Why can we not predict daily stock prices?"; "Why do large-scale pandemics strike periodically?". However, no one can resolve the above questions using chaos theory.

\subsection{What Are Systems Thinking?}

Generally, scientists classify physical phenomena into [logical problems] and [systematic problems]. In addition, this article has been described that the paradigm of scientific thinking is classified inti \{logical thinking\} and \{systems thinking\} as mentioned above. Unfortunately, classical physicists in the $17^{\text {th }}$ century approached it based on logical thinking with macroscopic static viewpoints in determinism, further, they have no analytical method for resolving circulation systems such as ecosystems as shown in Figure 1(b); they have no choice. Therefore, all scientists have no idea on the paradigm of systems thinking.

However, a new analytical theory has emerged in the $20^{\text {th }}$ century, despite of, sticking to primitive theories and rejecting new solutions is no different from medieval astronomers who excluded the heliocentric theory. Otherwise, we must be proficient in both systems thinking and logical thinking. The author would like to present briefly mathematical background for the readers here.

(Mathematical Background) Classical physicists have understood all nonoilier 
dynamic systematic problems (see Figure 1(b)) as the black box shown in Figure $1(\mathrm{c})$, and they are resolved it using logical solutions such as algebra and statistics based on logical thinking; it is in their academic framework. However, this is a serious misunderstanding. While all dynamic physical phenomena in nature must be defined it as closed loop systems as shown in Figure 1(c), including open loop systems. However, all scientists keep in mind that its internal structure and mechanism is as shown in Figure 2. In this case we need to know the characteristics. First, it must be approached with microscopic dynamic viewpoint based on systems thinking, because the output of systems is fluctuated endless as if complexity; moreover, it will be converged to steady state by selfcontrolling. Therefore, the systems thinking is likened the optimizing process. Hence, it is not difficult discipline. Thus, physicists need to study though interdisciplinary science.

For instance, we can transform the dynamic systems such as food chains in Figure 1(b) into closed loop system with feedback as shown in Figure 1(c); it is compatible to each other. And then, its mechanism of food chain is as follow. It can be transformed into a closed-loop system with feedback as shown in Figure 1 (c) and Figure 2; it has two elements such as predator $\mathrm{Q}(\mathrm{s})$ and prey $\mathrm{H}(\mathrm{s})$ [where $s$ is the Laplace operator]. If each element is related inversely with each other, it's time chart is presented in Figure 4(a). In this case, we can analyze the ecosystem through Figure 2. As the result, the numbers of predators or prey can predict its trend; it repeatedly increases and decreases endlessly according to time; further, converge to equilibrium or zero. Surprisingly, it can be explained and proved the mechanism via experiment using above mentioned simulator. [Remark; it is similar to Invisible Hand.] In this case, we can define its parameter $\mathrm{F}(\mathrm{s})$ of food chain is Equation (1).

$$
F(s)=\frac{\text { output } Y(s)}{\text { input } U(s)}=\frac{G(s)}{1 \mp G(s) H(s)}
$$

If we have the parameters of $\mathrm{Q}(\mathrm{s})$ and $\mathrm{H}(\mathrm{s})$ in Equation (1), we can determine the output in real time; it is a time series function. It can be displayed it by screen in simulator as shown in Figure 3. as the readers note it. Amazingly, it has contained complexity including irregularity, regularity, self-organization, and initial phenomenon. It means that nonlinear dynamics is impossible to resolve via logical thinking based in traditional physics; this study would like to prove why nonlinear dynamics cannot be resolved based on logical thinking using two books about complexity.

One is [Simply Complexity], written by a statistical physicist Neil Johnson [7], and the other is [Entropy], written by an economist Jeremy Rifkin [8]. They have approached nonlinear dynamics involving complexity based on logical thinking with static microscopic viewpoint what they have known. Therefore, they found only the external behavior of complexity such as irregularity, regularity, self-organization, and initial phenomenon. 


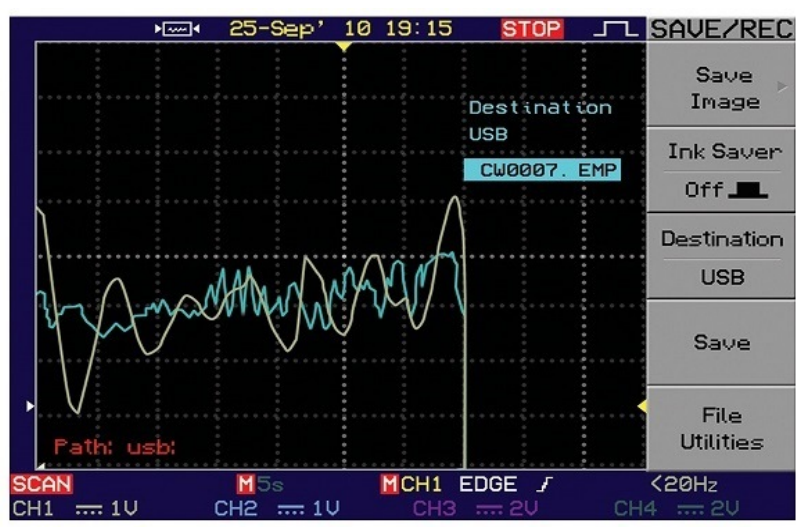

Figure 3. The typical behavior of output of closed loop systems with feedback if the input is random function. (blue line is input source, yellow line is output product.).

Nevertheless, they cannot find out other characteristics such as the output have no runaway and no overflow and no explosive. Paradoxically, if they knew this new solution, they would not have written the books on complexity.

\subsection{Overview of Analytical Method}

This section describes how to resolve nonlinear dynamics mathematically. First of all, we need to modeling the nonlinear dynamics systems such as food chain in ecosystems into closed loop systems with feedback as shown in Figure 1(c) and transforms into Figure 2. However, it is very difficult to be analyzed Equation (1) by hand. So, it must be resolve using an excellent computer program MATLAB [6], which does not require calculation by hand. In addition, there is prepared the analog simulator in Figure 2 in right side, which is able to reproduce the nonlinear dynamics in real time under virtual circumstances. Amazingly, it can be resolved by anyone who are not know systems analyzing; moreover, it can be simulated all types of closed loop systems in natural world. Hence, if physicists avoid experimenting with the above device, they are fooling; moreover, self-deception.

Next, there is a very important issue as follows if anyone wish to resolve their unsolved nonlinear dynamic problems. They should be followed following steps: [modeling-simulation-verification-return]. Since all closed-loop systems are impossible to calculate in reversely because it has no reversibility as inherent characteristics. Thus, nonlinear dynamics systems within closed loop systems as shown in Figure 2 must be solved repeatedly such as circulation analysis; it has no choice. As the result, the readers obtain the output of system as Equation (2).

$$
y(t)=1-A \cdot \mathrm{e}^{-B \cdot t} \sin (W \cdot t+\varphi)
$$

where $t$ is time, and $A, B, W$, and $\varphi$ are variable constants. The readers should note that Equation (2), which is a time series function. Amazingly, the Equation (2) has complexity including irregularity, regularity, self-organization, initial phenomenon. Therefore, Figure 3 is displayed to overlapped complexity with random input. Thus, the author encourages to conform the result using the si- 
mulation device as shown in Figure 2. Hence, nonlinear dynamics are solvable problems.

\subsection{Application Examples}

In this study, the new solution for resolving nonlinear dynamics based on systems thinking, which is resolved by a non-physicist on behalf of a physicist for the first time. Moreover, it was resolved and achieved via interdisciplinary research. However, most physicists remained as outsiders because do not welcome it. Moreover, many physicists have a doubt this solution, this section will resent four application examples to physicists; it is perfect evidence that the new solution is more advanced that the old chaos theory. There are the butterfly effect, random walk, logistic curve, and Kuhn's innovation theory as below.

\subsubsection{Redefined Lorenz's Butterfly-Effect}

Meanwhile, it was first claimed in 1963 by Edward Norton Lorenz [9], who was not a physicist but a meteorologist. Observing that a small numerical variation $(0.506127>0.5016)$ during an iterative calculation process in a weather forecast led to a completely different result, he named it the butter-fly effect, which appealed to the public and welcomed by determinists. Unfortunately, the author concluded that it was misunderstanding as the initial phenomenon, as shown in Figure 4(c).

(Modeling) However, Lorenz made a serious mistake such as assuming that atmospheric entropy increases infinitely without saturation. For instance, the heat energy from the sun $\mathrm{Q}(\mathrm{s})$ is moved to the Polar region and exhausted to space based on the law of energy conservation; it is defined as $\mathrm{H}(\mathrm{s})$. As the result, the heat circulation systems can transform into a basic model system based on systems thinking, as shown in Figure 2. It is not explosive, overflow and runaway absolutely.

(Simulation) Any scientist can analyze it using MATLAB. If the initial phenomenon is rapidly increasing (overshooting) in Figure 3(c), it similar to the butterfly effect. For instance, the same as numerous disasters in history, such as the Great Depression, World War, and Financial Crisis, it have occurred, but it is restored to the original state within a short period as shown in Figure 3(b).

(Verification) In conclusion, the butterfly effect is the result of exaggerated and expanded interpretation. Non-physicists have no reason to trust Lorenz no more. Paradoxically, if Lorenz had known the reason, he would not have made the assertion. Thus, this study classifies his assertion as fiction, which must be immediately eliminated from modern physics.

\subsubsection{Redefined Random Walk in Stock Market}

(Hypothesis) Physicists in Wall Street studied about the prediction of stock and they defined it as a random walk [10]. If the positive entropy of the seller desiring to sell at a high price is defined as $\mathrm{Q}(\mathrm{s})$ and the negative entropy of the buyer desiring to buy at a low price is defined as $\mathrm{H}(\mathrm{s})$, we can build model it as a closed 
(a)

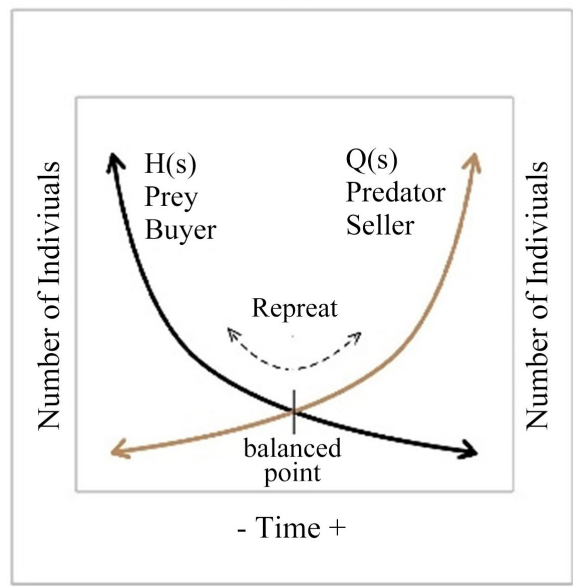

(d)

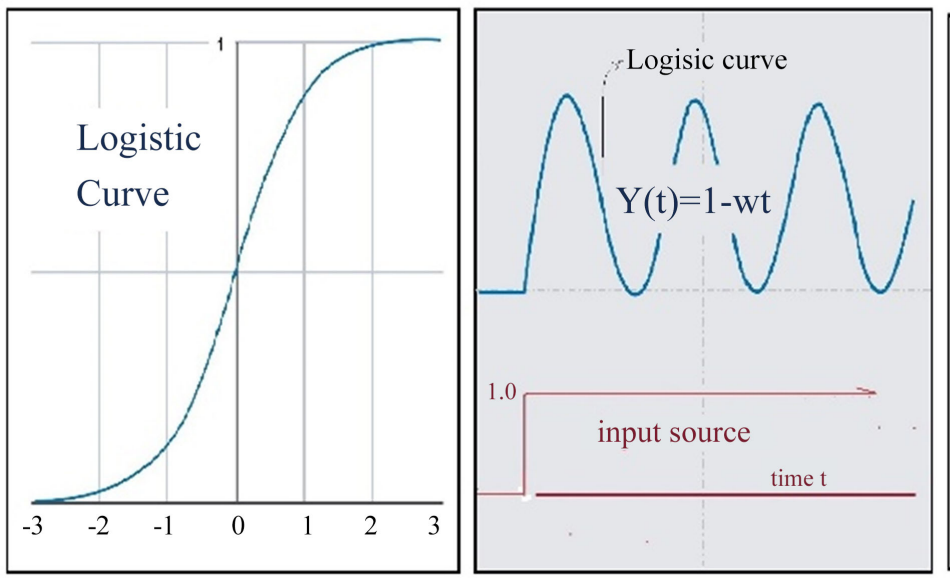

(c)

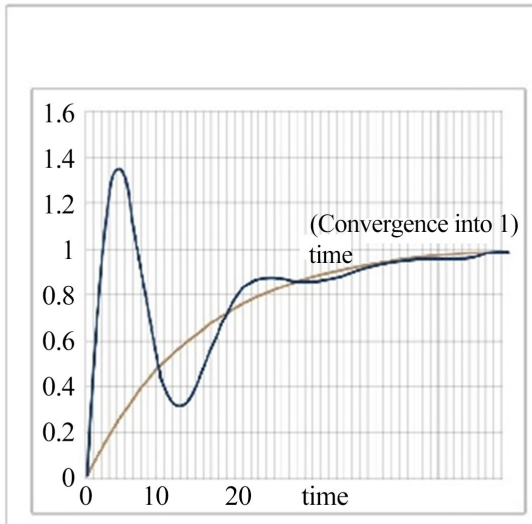

(f)

Figure 4. (a) A time chart of food chain or stock market; (b) Response of impulse (accident); (c) An example of initial phenomenon in complexity; (d) Logistic curve; (e) Analyzing result of logistic curve; (f) Time chart of Kuhn's innovation theory.

loop system as shown in Figure 2; it is the same as internal structure of stock market. If there is no incoming information of stock price, its output is converged in zero.

(Simulation) The above hypothesis can be easily simulated using MATLAB or a specially designed analog simulator (see Figure 2 right side). In this case, the behavior of output is determined as Equation (2); it is fluctuated endless. Thus, If the stock information is flow in stock market, the behavior of output as shown in Figure 3. It is typical behavior of complexity. Unfortunately, they misunderstand the result, so they named it as Random Walk.

(Verification) As the result, random walk is a time-series function. Paradoxically, if physicists have known the new solution, they do make the random walk.

\subsubsection{Redefined Verhulst's Logistic Curve}

(Hypothesis) It was asserted by the mathematician Verhulst [11], and it tries to define again why the increasing and decreasing population follows the sigmoid $S$ curve, as shown in Figure 3(d). It can be proven his assertion through the food chain in an ecological system. The number of predators and prey in the food 
chain are defined as $\mathrm{Q}(\mathrm{s})$ and $\mathrm{H}(\mathrm{s})$, respectively, in Figure 2.

(Simulation) In this case, the output of ecosystem is provided Equation (2) above. Based on this equation, we can resolve and obtain the following equation $y(t)=1-\sin (\omega t)$. It is a periodic sine curve, as shown in Figure 3(e). Therefore, the populations continue to increase and decrease repeatedly to along the $S$ curve.

(Verification) if anyone draws the above equation without dimensions, we can obtain the sigmoid $\mathrm{S}$ as shown in Figure 3(d), thus, the logistic curve is a time series function. Paradoxically, Verhulst has known the above systematic solution, he would not have made the assertion.

\subsubsection{Tomas Kuhn's Scientific Revolution Structure}

Kuhn's innovation theory is a famous philosophical principle that is established in modern science. He asserted the structure of scientific revolution with following sequences: scientific innovation-paradigm shift-normal science.

(Modeling) We need to redefine it. All dynamic problems within closed-loop systems in nature are time series functions. For instance, if the production cost of invention such as weapons or mobiles $\mathrm{Q}(\mathrm{s})$ and the purchasing ability $\mathrm{H}(\mathrm{s})$, we can define as a closed loop systems such as Figure 2; in this case, it will be slowly increasing and converge into saturation along equation $y(t)=1-e^{-x t}$ (where $\mathrm{x}$ is constant) based on Equation (2). An then, its timing chat is shown in Figure 3(f), which have three section such as innovation, paradigm shift and saturation (normal science). In other words, it gradually increases by time, finally, it is saturated state as shown in Figure 3(c).

(Simulation and Verification): As an examples, steam engine, gun powder, fertilizer, semiconductors, internet, and mobile phone. Mobile technology developed slowly for 150 years with the following sequence: invention-paradigm shift-normal science as shown in Figure 3(f). Hence, it out of philosophical problems. Paradoxically, he had known the new solution, he would not have made the assertion.

\section{Result}

1) There are many unsolved nonlinear dynamics problems in science, physicists have resolved these problems using as chaos theory in statistical physics, which is logical solution based on logical thinking. However, it is a serious mistake.

2) Because, all systematical problem should be analyzed using systematic solution based on systems thinking, however, the chaos theory is not systematic solution but it is solved by algebra and statistics. Therefore, chaos theory is imperfect.

3) In 2021, non-physicist on the behalf of physicist succeeded in resolving the problems based on the systems thinking. Regrettably, physicists unwelcome it because it is like Copernican theory. And the, physicists themselves are remained outsiders. 
4) However, there is no problem. Non-physicists need not to cooperate with physicists because it is nothing to do with them. Moreover, non-physicists have no reason to follow them unless clarify their shortcoming in their discipline

\section{Discussions}

This article would be a great achievement, like the Copernican theory, in modern science. Since it would be resolved unsolved nonlinear dynamics problems based on systems thinking via interdisciplinary science. Moreover, it presents solutions achieved by non-physicists on behalf of physicists, as shown in Table 1. Unfortunately, physicists yet remain outsiders who expected it to fail. Why do they so? If the above solution is true, determinists will be damaged from outside; they would be lost their reliability as did medieval astronomers. Otherwise, if it is false, there is no problem happened. Hence, non-physicists already have known what they want. They want to maintain the existing academic framework of determinism.

Paradoxically, if most determinists want to remain outsiders who want to stay safe zone, we need to exclude determinists from the new solution because they have nothing to do with other scientists and the public. It must be studied by non-physicists on behalf of physicists in the future because they have no ability to review and verify the new solution in Table 1 . Regrettably, it is very disadvantageous to them, such as medieval heliocentric theory. Again, non-physicists and the public in the world would understood their shortcomings in the Ptolemaic theory; moreover, untrust them as if medieval astronomers.

Meanwhile, this article prepared perfect evidence that the chaos theory is imperfect solution involving mysticism, moreover, also how to prove and verify it through experiment using devices. These evidence as application examples present in Section 2.5; butterfly-effect; random walk; logistic curve; Kuhn's innovation theory based on systems thinking. If determinists cannot understand it, they have no qualification to evaluate this article.

On the other hand, if they want to remained outsiders, non-physicists need not cooperate with them. In this case, non-physicists need to find other way. For instance, we have to separate the new solution from physics. With the new solution, non-physicists acting on behalf of physicists can cover independently many unsolved nonlinear dynamics problems and metaphysical problems in all sciences. If so, other scientists will have to redefine their statistical physics. For instance, examination of Lorenz's butterfly effect will reveal that it is a mysterious problem without an academic background, but a time series function, as shown in Figure $4(\mathrm{c})$. Hence, other scientists need to learn the analyzing theory in engineering.

In addition, the author wishes to establish the above solutions in modern science. Thus, we propose to apply to a well-known research organization, the Santa Fe Institute [12], in the USA. The SFI's mission is to resolve complexity in economics and ecology based on logical thinking by physicists on behalf of hu- 
manities scholars. However, they cannot fulfill their mission. For instance, they approach systematic problems, such as macroeconomics and ecosystems, based on logical thinking. this is a mistake because it is like driving a horse cart instead of an engine-driven truck. Paradoxically, if they knew the new solution based on the systems thinking, they would already have succeeded in resolving their mission.

\section{Conclusions}

This article presents the detailed new systems solution in Section 2, which has been achieved by non-physicists based on systems thinking on behalf of physicists, for the first time. Nevertheless, do not welcome it because it is like medieval Copernican theory, further, they already have solution such as chaos theory in their physics. So, physicists do not accept it; moreover, they remain as outsiders who wish the author to fail; it does not make sense.

However, the old chaos theory is not perfect but the new solution is more advanced and precise and easy solution than the old chaos theory. It can be proved using experiment. So, this article present perfect evidence in Section 2.5. However, if physicists remain outsiders, other scientists need not follow them. This new solution will be applied to all science and established in modern science.

In conclusion, the author expects to apply the above new solution to all around scientific discipline. Thus, this study contributes to advances in science. Hence, the author encourages scientists to learn control theory.

\section{Acknowledgements}

Eho technology co. research center supported research.

\section{Conflicts of Interest}

The authors declare no conflicts of interest regarding the publication of this paper.

\section{References}

[1] Casti, J.L. (1995) Complexification: Explaining a Paradoxical World through the Science of Surprise. Harper Perennial, New York.

[2] Golnaraghi, F. and Kuo, B.C. (1998) Automatic Control Systems. 10th Edition, McGraw-Hill Education, New York

[3] Cha, D.S. (2015) Establishment of New Solution for Complex Systems in Multidisciplinary Science Based on Feedback System Analysis Method and Proven by Simulator. Journal of Modern Physics, 6, 1927-1934.

https://www.scirp.org/journal/paperinformation.aspx?paperid=60738 https://doi.org/10.4236/jmp.2015.613198

[4] Cha, D. and Jun, H. (2020) The Origin of Nonlinear Dynamics Involving Complexity in Modern Sciences. Open Journal of Applied Sciences, 10, 654-662. https://www.scirp.org/journal/paperinformation.aspx?paperid $=103755$ https://doi.org/10.4236/ojapps.2020.1010045

[5] Goodman, M. (2018) Systems Thinking: What, Why, When, Where, and How? 
https://thesystemsthinker.com/systems-thinking-what-why-when-where-and-how/

[6] MATLAB Simulink (2021) MathWorks Co.

https://kr.mathworks.com/matlabcentral/?s tid=gn mlc

[7] Neil, J. (2020) Simply Complexity (2007 Korea Edition). Bada Publishing, Seoul.

[8] Jeremy, R. (1981) Entropy: A New World View. Bantam Books, London.

[9] Lorenz, E. (1963) Deterministic Non periodic Flow. JAS, 20.

https://journals.ametsoc.org/view/journals/atsc/20/2/1520-0469 $1963 \quad 020 \quad 0130 \mathrm{dn}$ f 20 co $2 . x m l$ https://doi.org/10.1175/1520-0469(1963)020<0130:DNF>2.0.CO;2

[10] Random Walk "Encyclopedia of Mathematics". https://encyclopediaofmath.org/wiki/Random walk

[11] Linacre, L.J. (2009) Why Logistic (Sigmoid) Ogive and Not Autocatalytic Curve? https://rasch.org/rmt/rmt64k.htm

[12] Santa Fe Institute (2021). https://santafe.edu/about 